\title{
On the Action of Riesz Transforms on the Class of Bounded Functions
}

\section{Adam Osȩkowski}

Received: 18 November 2012 / Accepted: 19 July 2013 / Published online: 3 August 2013

(C) The Author(s) 2013. This article is published with open access at Springerlink.com

Abstract The paper is devoted to the $d$-dimensional extension of the classical identity of Stein and Weiss concerning the action of the Hilbert transform on characteristic functions. Let $\left(R_{j}\right)_{j=1}^{d}$ be the collection of Riesz transforms in $\mathbb{R}^{d}$. For $1 \leq p<\infty$, we determine the least constants $c_{p, d}, C_{p, d}$ such that

$$
\begin{array}{r}
\int_{\mathbb{R}^{d}} f(x)\left|R_{j} f(x)\right|^{p} \mathrm{~d} x \leq c_{p, d}\|f\|_{L^{1}\left(\mathbb{R}^{d}\right)}, \\
\int_{\mathbb{R}^{d}}(1-f(x))\left|R_{j} f(x)\right|^{p} \mathrm{~d} x \leq C_{p, d}\|f\|_{L^{1}\left(\mathbb{R}^{d}\right)}
\end{array}
$$

for any Borel function $f: \mathbb{R}^{d} \rightarrow[0,1]$. The proof rests on probabilistic methods and the construction of appropriate harmonic functions on $[0,1] \times \mathbb{R}$.

Keywords Hilbert transform - Riesz transform - Martingale - Differential subordination $\cdot$ Re-expansion operator $\cdot$ Best constants

Mathematics Subject Classification (2010) Primary 42B15 - 42B20 • 46E30; Secondary 60G44

Communicated by F. Colombo.

A. Osȩkowski $(\bowtie)$

Department of Mathematics, Informatics and Mechanics,

University of Warsaw, Banacha 2, 02-097 Warsaw, Poland

e-mail: ados@mimuw.edu.pl 


\section{Introduction}

The Hilbert transform $\mathcal{H}$ on the line is the operator defined by the principal value integral

$$
\mathcal{H} f(x)=\frac{1}{\pi} \int_{\mathbb{R}} \frac{f(x-y)}{y} \mathrm{~d} y .
$$

Let $E \subset \mathbb{R}$ be a measurable set of finite Lebesgue measure. A classical result of Stein and Weiss [19] asserts that the distribution function of $\mathcal{H} \chi_{E}$ depends only on the measure of $E$ and is non-sensitive to the inner structure of $E$. Precisely, for any $t>0$ we have the identity

$$
\left|\left\{x \in \mathbb{R}:\left|\mathcal{H} \chi_{E}(x)\right|>t\right\}\right|=\frac{2|E|}{\sinh (\pi t)} .
$$

This fact can be proved using various tools; for different approaches, consult Stein and Weiss [19,20], Calderón [5] and the two recent papers: [6] by Colzani et al. and [14] by Laeng. In fact, in the latter paper a stronger statement, concerning the behavior of $\mathcal{H} \chi_{E}$ restricted to $E$ and $\mathbb{R} \backslash E$, was established. Namely, it was shown that for any $t>0$,

$$
\left|\left\{x \in E:\left|\mathcal{H} \chi_{E}(x)\right|>t\right\}\right|=\frac{2|E|}{e^{\pi t}+1}
$$

and

$$
\left|\left\{x \in \mathbb{R} \backslash E:\left|\mathcal{H} \chi_{E}(x)\right|>t\right\}\right|=\frac{2|E|}{e^{\pi t}-1} .
$$

To accomplish this, Laeng proved that

$$
\int_{E}\left|\mathcal{H} \chi_{E}(x)\right|^{p} \mathrm{~d} x=2 \int_{0}^{\infty} \frac{p t^{p-1}}{e^{\pi t}+1} \mathrm{~d} t \cdot|E|, \quad 1 \leq p<\infty,
$$

and

$$
\int_{\mathbb{R} \backslash E}\left|\mathcal{H} \chi_{E}(x)\right|^{p} \mathrm{~d} x=2 \int_{0}^{\infty} \frac{p t^{p-1}}{e^{\pi t}-1} \mathrm{~d} t \cdot|E|, \quad 1<p<\infty,
$$

and combined it with the fact that any two functions $f, g$, which have the same $p$-th norms for $p$ lying in some interval ( $\left.p_{1}, p_{2}\right)$, are equidistributed (cf. [6]).

The principal goal of this paper is to provide another proof of the identities (1.5) and (1.6), with the use of probabilistic methods: the identities will be deduced from their novel counterparts in martingale theory. In fact, it will allow us to study the more general question concerning the action of the Hilbert transform on the class of bounded 
functions. Our approach easily leads to appropriate higher-dimensional results, to formulate which we require some additional background. Suppose that $d \geq 1$ is a given integer. The counterpart of the Hilbert transform in $\mathbb{R}^{d}$ is the collection of Riesz transforms $\left(R_{j}\right)_{j=1}^{d}$ (see e.g. Stein [18]). This family of singular integral operators is given by

$$
R_{j} f(x)=\frac{\Gamma\left(\frac{d+1}{2}\right)}{\pi^{(d+1) / 2}} \int_{\mathbb{R}^{d}} \frac{x_{j}-y_{j}}{|x-y|^{d+1}} f(y) \mathrm{d} y, \quad j=1,2, \ldots, d .
$$

Here the integrals, as in (1.1), are supposed to exist in the sense of Cauchy principal values. When $d=1$, the collection consists of only one element, the Hilbert transform on the line. There is an alternative definition of $\left(R_{j}\right)_{j=1}^{d}$, as the family of Fourier multipliers with the symbols $\left(-i \xi_{j} /|\xi|\right)_{j=1}^{d}, \xi \in \mathbb{R}^{d} \backslash\{0\}$. That is, we have the following relation between the Fourier transforms of $f$ and $R_{j} f$ :

$$
\widehat{R_{j} f}(\xi)=-\frac{\xi_{j}}{|\xi|} \hat{f}(\xi), \quad \text { for } \xi \in \mathbb{R}^{d} \backslash\{0\} .
$$

We are ready to formulate the main result.

Theorem 1.1 Let $d \geq 1$ be a given integer and suppose that $f$ is a Borel function on $\mathbb{R}^{d}$ taking values in the interval $[0,1]$. Then for any $j \in\{1,2, \ldots, d\}$ we have

$$
\int_{\mathbb{R}^{d}} f(x)\left|R_{j} f(x)\right|^{p} d x \leq 2 \int_{0}^{\infty} \frac{p t^{p-1}}{e^{\pi t}+1} d t \cdot\|f\|_{L^{1}\left(\mathbb{R}^{d}\right)}, \quad 1 \leq p<\infty,
$$

and

$$
\int_{\mathbb{R}^{d}}(1-f(x))\left|R_{j} f(x)\right|^{p} d x \leq 2 \int_{0}^{\infty} \frac{p t^{p-1}}{e^{\pi t}-1} d t \cdot\|f\|_{L^{1}\left(\mathbb{R}^{d}\right)}, \quad 1<p<\infty
$$

Both inequalities are sharp for each $j$ and $d$. They are already sharp if $f$ is assumed to run over the class of characteristic functions of measurable sets.

Here by sharpness we mean that neither of the constants $2 \int_{0}^{\infty} \frac{p t^{p-1}}{e^{\pi t}+1} \mathrm{~d} t, 2 \int_{0}^{\infty} \frac{p t^{p-1}}{e^{\pi t}-1} \mathrm{~d} t$ in (1.8) and (1.9) can be replaced by a smaller number. One easily shows (cf. [14]) that these constants can be expressed in terms of Gamma and Riemann zeta functions: we have

$$
2 \int_{0}^{\infty} \frac{p t^{p-1}}{e^{\pi t}+1} \mathrm{~d} t=\frac{1}{\pi^{p}}\left(2-\frac{1}{2^{p-2}}\right) \Gamma(p+1) \zeta(p)
$$

(when $p=1$, we take the limit: $2 \log 2 / \pi$ ) and 


$$
2 \int_{0}^{\infty} \frac{p t^{p-1}}{e^{\pi t}-1} \mathrm{~d} t=\frac{2}{\pi^{p}} \Gamma(p+1) \zeta(p)
$$

If we put $f=\chi_{E}$ for a given Borel subset $E \subset \mathbb{R}^{d}$ with $|E|<\infty$, we obtain the following $d$-dimensional analogues of (1.5) and (1.6): for $1 \leq j \leq d$,

$$
\begin{array}{r}
\int_{E}\left|R_{j} \chi_{E}\right|^{p} \mathrm{~d} x \leq 2 \int_{0}^{\infty} \frac{p t^{p-1}}{e^{\pi t}+1} \mathrm{~d} t \cdot|E|, \\
\int_{\mathbb{R}^{d} \backslash E}\left|R_{j} \chi_{E}\right|^{p} \mathrm{~d} x \leq 2 \int_{0}^{\infty} \frac{p t^{p-1}}{e^{\pi t}-1} \mathrm{~d} t \cdot|E| .
\end{array}
$$

Adding these estimates, we get the inequality

$$
\int_{\mathbb{R}^{d}}\left|R_{j} \chi_{E}\right|^{p} \mathrm{~d} x \leq 2 \int_{0}^{\infty} \frac{p t^{p-1}}{\sinh (\pi t)} \mathrm{d} t \cdot|E|, \quad 1<p<\infty .
$$

We will show that this estimate is also sharp, for any values of $d$ and $j$.

Let us mention here two interesting related questions. First, can the Riesz transform $R_{j}$ be replaced in (1.8), (1.9) and (1.10) by its vector version $R=\left(R_{1}, R_{2}, \ldots, R_{d}\right)$ with no change in the constant? (We interpret $|R f(x)|$ as $\left(\sum_{j=1}^{d}\left|R_{j} f(x)\right|^{2}\right)^{1 / 2}$.) Unfortunately, we have been unable to shed any light on this problem with the methods developed here. The second question, which we also did not manage to answer, concerns the $d$-dimensional version of (1.3) and (1.4). This is closely related to a longstanding open problem of Stein concerning the weak-type $(1,1)$ inequality for Riesz transforms: it is not known whether this estimate holds with a constant independent of the dimension.

The above results can be applied to the study of the so-called re-expansion operator. Let $\mathcal{F}_{c}$ and $\mathcal{F}_{s}$ be the cosine and sine Fourier transforms on $\mathbb{R}_{+}$, respectively. That is, for $x>0$ and any Borel function $f$ on $\mathbb{R}_{+}$,

$$
\mathcal{F}_{c} f(x)=\sqrt{\frac{2}{\pi}} \int_{\mathbb{R}_{+}} f(t) \cos t x \mathrm{~d} t, \quad \mathcal{F}_{s} f(x)=\sqrt{\frac{2}{\pi}} \int_{\mathbb{R}_{+}} f(t) \sin t x \mathrm{~d} t .
$$

Both $\mathcal{F}_{c}$ and $\mathcal{F}_{S}$ are unitary and self-adjoint operators on $L^{2}\left(\mathbb{R}_{+}\right)$. We define the reexpansion operator $\Pi$ on $\mathbb{R}_{+}$by the identity $\Pi=\mathcal{F}_{s} \mathcal{F}_{c}$. This operator is interesting from the analytical point of view, as the object of spectral analysis and also appears naturally in the scattering theory. For more on the subject, consult Birman [3], Gokhberg and Krupnik [8] and Il'in [12,13].

The question about various norms of $\Pi$ has gathered some interest in the literature. Hollenbeck et al. [11] proved that the re-expansion operator has the same $p$-th norm 
as the Hilbert transform: $\|\Pi\|_{L^{p}\left(\mathbb{R}_{+}\right) \rightarrow L^{p}\left(\mathbb{R}_{+}\right)}=\|\mathcal{H}\|_{L^{p}(\mathbb{R}) \rightarrow L^{p}(\mathbb{R})}$ for $1<p<\infty$. Then it was shown by the author in [15] that the weak $p$-th norms of $\Pi$ and $\mathcal{H}$ coincide for $1 \leq p \leq 2$ : $\|\Pi\|_{L^{p}\left(\mathbb{R}_{+}\right) \rightarrow L^{p, \infty}\left(\mathbb{R}_{+}\right)}=\|\mathcal{H}\|_{L^{p}(\mathbb{R}) \rightarrow L^{p, \infty}(\mathbb{R})}$. We continue this line of research and study the action of $\Pi$ on $L_{\infty}^{+}$.

Theorem 1.2 Suppose that $f: \mathbb{R}_{+} \rightarrow[0,1]$ is a given function. Then for any $p \in$ $[1, \infty)$ we have

$$
\int_{\mathbb{R}_{+}} f(x)|\Pi f(x)|^{p} d x \leq 2 \int_{0}^{\infty} \frac{p t^{p-1}}{e^{\pi t}+1} d t \cdot\|f\|_{L_{1}\left(\mathbb{R}_{+}\right)}
$$

and

$$
\int_{\mathbb{R}_{+}}(1-f(x))|\Pi f(x)|^{p} d x \leq 2 \int_{0}^{\infty} \frac{p t^{p-1}}{e^{\pi t}-1} d t \cdot\|f\|_{L_{1}\left(\mathbb{R}_{+}\right)} .
$$

\section{Both inequalities are sharp.}

This result can be proved with the use of a well-known connection between the inequalities for re-expansion operator and the estimates for Riesz transforms and martingales. We omit the details, referring the interested reader to $[11,15,16]$.

A few words about the organization of the paper. Our first step is to establish appropriate martingale inequalities, the probabilistic analogues of (1.8) and (1.9): see the next section. Then, in Sect. 3, we exploit a certain version of the so-called "background radiation process" of Gundy and Varopoulos [10], which enables us to deduce (1.8) and (1.9). The final part of the paper is devoted to the second part of Theorem 1.1, i.e., the optimality of the constants.

\section{Martingale Inequalities}

As mentioned in the Introduction, the results of this paper depend heavily on appropriate martingale inequalities. We start with introducing the necessary probabilistic background and notation. Let $(\Omega, \mathcal{F}, \mathbb{P})$ be a complete probability space, filtered by $\left(\mathcal{F}_{t}\right)_{t \geq 0}$, a nondecreasing family of sub- $\sigma$-fields of $\mathcal{F}$, with the property that $\mathcal{F}_{0}$ contains all the events of probability 0 . Assume that $X, Y$ are two adapted real-valued martingales with right-continuous paths that have limits from the left. We will denote by $[X, Y]$ the quadratic covariance process of $X$ and $Y$, see e.g. Dellacherie and Meyer [7] for details. The martingales $X, Y$ are said to be orthogonal if $d[X, Y]=0$ almost surely (that is, the process $[X, Y]$ is constant with probability 1). Following Bañuelos and Wang [1] and Wang [21], we say that $Y$ is differentially subordinate to $X$, if the process $\left([X, X]_{t}-[Y, Y]_{t}\right)_{t \geq 0}$ is nonnegative and nondecreasing as a function of $t$. As an example, assume that $B$ is a $d$-dimensional Brownian motion, $H$ is a predictable 
process with values in $\mathbb{R}^{d}$ and $A$ is a $d \times d$ matrix such that $\langle A x, x\rangle=0$ and $|A x| \leq|x|$ for any $x \in \mathbb{R}^{d}$. If we put

$$
X_{t}=\int_{0}^{t} H_{s} \mathrm{~d} B_{s}, \quad Y_{t}=\int_{0}^{t} A H_{s} \mathrm{~d} B_{s}, \quad t \geq 0,
$$

then $Y$ is orthogonal and differentially subordinate to $X$. This follows immediately from the identities

$$
[X, Y]_{t}=\int_{0}^{t}\left\langle A H_{s}, H_{s}\right\rangle \mathrm{d} s
$$

and

$$
[X, X]_{t}-[Y, Y]_{t}=\int_{0}^{t}\left(\left|H_{s}\right|^{2}-\left|A H_{S}\right|^{2}\right) \mathrm{d} s .
$$

The differential subordination implies many interesting inequalities comparing various sizes of $X$ and $Y$. The literature on this subject is quite extensive and it is not possible to review it here; we refer the reader to the survey [4] by Burkholder, the paper of Wang [21] and the monograph [17] by the author. Here we only mention one result, of Bañuelos and Wang [21], to be needed later. We use the notation $\|X\|_{p}=\sup _{t \geq 0}\left\|X_{t}\right\|_{p}$ for $1 \leq p \leq \infty$.

Theorem 2.1 Suppose that $X, Y$ are orthogonal martingales such that $Y$ is differentially subordinate to $X$. Then for any $1<p<\infty$,

$$
\|Y\|_{p} \leq \cot \left(\frac{\pi}{2 p^{*}}\right)\|X\|_{p},
$$

where $p^{*}=\max \{p, p /(p-1)\}$. The constant is the best possible.

The main result of this section is the following.

Theorem 2.2 Suppose that $X, Y$ are orthogonal martingales such that $X$ takes values in $[0,1], Y$ is differentially subordinate to $X$ and $Y_{0} \equiv 0$. Then for any $1 \leq p<\infty$,

$$
\mathbb{E} X_{\infty}\left|Y_{\infty}\right|^{p} \leq \frac{1}{\pi} \int_{0}^{\infty} \frac{\left|\frac{1}{\pi} \log s\right|^{p} \sin \left(\pi\|X\|_{1}\right)}{s^{2}+1+2 s \cos \left(\pi\|X\|_{1}\right)} d s
$$

and, for $1<p<\infty$,

$$
\mathbb{E}\left(1-X_{\infty}\right)\left|Y_{\infty}\right|^{p} \leq \frac{1}{\pi} \int_{-\infty}^{0} \frac{\left|\frac{1}{\pi} \log (-s)\right|^{p} \sin \left(\pi\|X\|_{1}\right)}{s^{2}+1+2 s \cos \left(\pi\|X\|_{1}\right)} d s .
$$


Both inequalities are sharp: for each of them there is a nontrivial pair $(X, Y)$ for which both sides are equal.

Here $X_{\infty}, Y_{\infty}$ stand for the pointwise limits of $X$ and $Y$ as $t \rightarrow \infty$; the existence of these limits follows at once from the boundedness of $X$ and $Y$ in $L^{2}$ (which is guaranteed by the condition $X \in[0,1]$ and Theorem 2.1 above).

The proof of Theorem 2.2 will be based on the existence of certain special harmonic functions. Let $H=\mathbb{R} \times(0, \infty)$ denote the upper half-space and let $S=[0,1] \times \mathbb{R}$ stand for the vertical strip in $\mathbb{R}^{2}$. Introduce the auxiliary functions $\mathcal{U}_{1}, \mathcal{U}_{2}: H \rightarrow \mathbb{R}$ by the Poisson integrals

$$
\mathcal{U}_{1}(\alpha, \beta)=\frac{1}{\pi} \int_{0}^{\infty} \frac{\beta\left|\frac{1}{\pi} \log t\right|^{p}}{(\alpha-t)^{2}+\beta^{2}} \mathrm{~d} t, \quad 1 \leq p<\infty
$$

and

$$
\mathcal{U}_{2}(\alpha, \beta)=\frac{1}{\pi} \int_{-\infty}^{0} \frac{\beta\left|\frac{1}{\pi} \log (-t)\right|^{p}}{(\alpha-t)^{2}+\beta^{2}} \mathrm{~d} t, \quad 1<p<\infty
$$

Obviously, $\mathcal{U}_{1}$ and $\mathcal{U}_{2}$ are harmonic on $H$ and satisfy

$$
\lim _{(\alpha, \beta) \rightarrow(z, 0)} \mathcal{U}_{1}(\alpha, \beta)= \begin{cases}0 & \text { for } z<0 \\ \left.\left(\frac{1}{\pi}\right)^{p}|\log | z\right|^{p} & \text { for } z>0\end{cases}
$$

and

$$
\lim _{(\alpha, \beta) \rightarrow(z, 0)} \mathcal{U}_{2}(\alpha, \beta)= \begin{cases}\left(\frac{1}{\pi}\right)^{p}|\log | z||^{p} & \text { for } z<0 \\ 0 & \text { for } z>0\end{cases}
$$

Consider a conformal mapping $\varphi(z)=-e^{-i \pi z}$ or, in real coordinates,

$$
\varphi(x, y)=\left(-e^{\pi y} \cos (\pi x), e^{\pi y} \sin (\pi x)\right)
$$

This function maps $(0,1) \times \mathbb{R}$ onto $H$. Define $U_{1}, U_{2}$ in the interior of the strip $S$ by the formula

$$
U_{i}(x, y)=\mathcal{U}_{i}(\varphi(x, y)), \quad i=1,2 .
$$

As the compositions of harmonic functions with conformal mappings, $U_{1}, U_{2}$ are harmonic on $(0,1) \times \mathbb{R}$ and, by (2.3) and (2.4), they can be extended to continuous functions on the whole $S$ using the equalities $U_{1}(0, y)=U_{2}(1, y)=0, U_{1}(1, y)=$ $U_{2}(0, y)=|y|^{p}$. Substituting $t=s e^{\pi y}$ in the integrals defining $\mathcal{U}_{1}$ and $\mathcal{U}_{2}$, we easily check that 


$$
U_{1}(x, y)=\frac{1}{\pi} \int_{0}^{\infty} \frac{\left|\frac{1}{\pi} \log s+y\right|^{p} \sin (\pi x)}{(s+\cos (\pi x))^{2}+\sin ^{2}(\pi x)} \mathrm{d} s
$$

and

$$
U_{2}(x, y)=\frac{1}{\pi} \int_{-\infty}^{0} \frac{\left|\frac{1}{\pi} \log (-s)+y\right|^{p} \sin (\pi x)}{(s+\cos (\pi x))^{2}+\sin ^{2}(\pi x)} \mathrm{d} s
$$

for $0<x<1$ and $y \in \mathbb{R}$. Further properties of $U_{1}, U_{2}$ are investigated in the lemma below.

Lemma 2.3 (i) The constants on right-hand sides of (2.1) and (2.2) are equal to $U_{1}\left(\|X\|_{1}, 0\right)$ and $U_{2}\left(\|X\|_{1}, 0\right)$, respectively.

(ii) Both $U_{1 x x}$ and $U_{2 x x}$ are nonpositive in the interior of $S$.

(iii) We have the majorizations

$$
U_{1}(x, y) \geq x|y|^{p} \quad \text { and } \quad U_{2}(x, y) \geq(1-x)|y|^{p}
$$

for any $(x, y) \in S$.

(iv) We have

$$
U_{1 y}(x, 0)=U_{2 y}(x, 0)=0 \quad \text { for } x \in(0,1) .
$$

Proof (i) This is a consequence of the formulas (2.6) and (2.7).

(ii) By the harmonicity of $U_{1}$ and $U_{2}$ inside the strip $S$, it suffices to show that $U_{1 y y}$ and $U_{2 y y}$ are nonnegative. But this is clear, in view of (2.6) and (2.7): for any $s \in \mathbb{R}$, the function $y \mapsto\left|\frac{1}{\pi} \log \right| s|+y|^{p}$ is convex.

(iii) By (ii), all we need is to show the majorization for $x \in\{0,1\}$. However, when $x=0$ or $x=1$, then both estimates in (2.8) become equalities.

(iv) It suffices to substitute $s:=1 / s$ in (2.6) and (2.7) to get that $U_{1}(x, y)=$ $U_{1}(x,-y)$ and $U_{2}(x, y)=U_{2}(x,-y)$ on $S$. This clearly yields the claim.

Later on, we will require the following technical fact, which appears, in a slightly different form, as Corollary 1 in Bañuelos and Wang [2].

Lemma 2.4 Suppose that $X, Y$ are real-valued orthogonal martingales such that $Y$ is differentially subordinate to $X$. Then $Y$ has continuous paths and is orthogonal and differentially subordinate to $X^{c}$, the continuous part of $X$.

We are ready to study the martingale inequalities (2.1) and (2.2).

Proof of Theorem 2.2 We will focus on the inequality (2.1), the second estimate (and its sharpness) can be shown in a similar manner. Fix $t \in(0, \infty)$ and introduce the process $\left(Z_{s}\right)_{s \geq 0}=\left(\left(X_{s}, Y_{s}\right)\right)_{s \geq 0}$. Since $U_{1}$ is of class $C^{\infty}$ in the interior of $S$, we may apply Itô's formula to obtain 


$$
U_{1}\left(Z_{t}\right)=U_{1}\left(Z_{0}\right)+I_{1}+\frac{1}{2} I_{2}+\frac{1}{2} I_{3}+I_{4}
$$

where

$$
\begin{aligned}
& I_{1}=\int_{0+}^{t} U_{1 x}\left(Z_{s-}\right) \mathrm{d} X_{s}+\int_{0+}^{t} U_{1 y}\left(Z_{s-}\right) \mathrm{d} Y_{s}, \\
& I_{2}=2 \int_{0+}^{t} U_{1 x y}\left(Z_{s-}\right) \mathrm{d}\left[X^{c}, Y\right]_{s}, \\
& I_{3}=\int_{0+}^{t} U_{1 x x}\left(Z_{s-}\right) \mathrm{d}[X, X]_{s}^{c}+\int_{0+}^{t} U_{1 y y}\left(Z_{s-}\right) \mathrm{d}[Y, Y]_{s}, \\
& I_{4}=\sum_{0<s \leq t}\left\{U_{1}\left(Z_{s}\right)-U_{1}\left(Z_{s-}\right)-U_{1 x}\left(Z_{s-}\right) \Delta X_{s}\right\} .
\end{aligned}
$$

Here $\Delta X_{s}$ denotes the jump of $X$ at time $s$. Note that we have used above the equalities $Y_{s-}=Y_{s}$ and $Y=Y^{c}$, which are due to the continuity of paths of $Y$. Let us analyze the above terms separately. We start from observing that $U_{1}\left(Z_{0}\right)=U_{1}\left(X_{0}, 0\right)=$ $U_{1}\left(\|X\|_{1}, 0\right)$. The term $I_{1}$ has zero expectation, since both stochastic integrals are martingales. Next, we have $I_{2}=0$, because of the orthogonality of $X^{c}$ and $Y$. The differential subordination together with Lemma 2.3 (ii) give

$$
I_{3} \leq \int_{0}^{t} U_{1 x x}\left(Z_{s-}\right) d[X, X]_{S}^{c}+\int_{0}^{t} U_{1 y y}\left(Z_{s-}\right) d[X, X]_{s}^{c}=0 .
$$

Finally, each summand in $I_{4}$ is nonpositive, by the concavity of $U_{1}(\cdot, y)$ for any fixed $y \in \mathbb{R}$ (again, apply Lemma 2.3 (ii)). Therefore, using the majorization of that lemma, we obtain the estimate

$$
\mathbb{E} X_{t}\left|Y_{t}\right|^{p} \leq \mathbb{E} U_{1}\left(X_{t}, Y_{t}\right) \leq U_{1}\left(\|X\|_{1}, 0\right)
$$

and (2.1) is established, in view of Fatou's lemma. To see that this estimate is sharp, pick any pair $X, Y$ of continuous-path orthogonal martingales satisfying $\mathrm{d}[X, X]=\mathrm{d}[Y, Y]$ and such that $X_{\infty}=\lim _{t \rightarrow \infty} X_{t} \in\{0,1\}$ with probability 1 . Then for any $t \geq 0$ we have $\mathbb{E} U_{1}\left(X_{t}, Y_{t}\right)=U_{1}\left(\|X\|_{1}, 0\right)$, so letting $t \rightarrow \infty$ gives

$$
\mathbb{E} X_{\infty}\left|Y_{\infty}\right|^{p}=\mathbb{E} U_{1}\left(X_{\infty}, Y_{\infty}\right)=U_{1}\left(\|X\|_{1}, 0\right),
$$

in view of Lebesgue's dominated convergence theorem. This proves the claim.

\section{Inequalities for Riesz Transforms in $\mathbb{R}^{d}$}

There is a well-known representation of Riesz transforms in terms of the so-called background radiation process, introduced by Gundy and Varopoulos in [10]. Let us 
briefly describe this connection. Throughout this section, $d$ is a fixed positive integer. Suppose that $X$ is a Brownian motion in $\mathbb{R}^{d}$ and let $Y$ be an independent Brownian motion in $\mathbb{R}$ (both processes start from the appropriate origins). For any $y>0$, introduce the stopping time $\tau(y)=\inf \left\{t \geq 0: Y_{t} \in\{-y\}\right\}$. For sufficiently regular $f$ (say, $f \in L^{p}\left(\mathbb{R}^{d}\right.$ ) for some $\left.1 \leq p<\infty\right)$, let $U_{f}: \mathbb{R}^{d} \times[0, \infty) \rightarrow \mathbb{R}$ stand for the Poisson extension of $f$ to the upper half-space. That is,

$$
U_{f}(x, y):=\mathbb{E} f\left(x+X_{\tau(y)}\right) .
$$

For any $(d+1) \times(d+1)$ matrix $A$ we define the martingale transform $A * f$ by

$$
A * f(x, y)=\int_{0+}^{\tau(y)} A \nabla U_{f}\left(x+X_{s}, y+Y_{s}\right) \cdot \mathrm{d}\left(X_{s}, Y_{s}\right) .
$$

Let us stress here that $A * f(x, y)$ is a random variable for each $x, y$. Now, for any $f \in C_{0}^{\infty}\left(\mathbb{R}^{d}\right)$, any $y>0$ and any matrix $A$ as above, define $\mathcal{T}_{A}^{y} f: \mathbb{R}^{d} \rightarrow \mathbb{R}$ using the bilinear form

$$
\int_{\mathbb{R}^{d}} \mathcal{T}_{A}^{y} f(x) g(x) \mathrm{d} x=\int_{\mathbb{R}^{d}} \mathbb{E}\left[A * f(x, y) g\left(x+X_{\tau(y)}\right)\right] \mathrm{d} x,
$$

where $g$ runs over $C_{0}^{\infty}\left(\mathbb{R}^{d}\right)$. Less formally, $\mathcal{T}^{y} f$ is given as the following conditional expectation with respect to the measure $\tilde{\mathbb{P}}=\mathbb{P} \otimes \mathrm{d} x$ ( $\mathrm{d} x$ denotes Lebesgue's measure on $\mathbb{R}^{d}$ ): for any $z \in \mathbb{R}^{d}$,

$$
\mathcal{T}_{A}^{y} f(z)=\tilde{\mathbb{E}}\left[A * f(x, y) \mid x+X_{\tau(y)}=z\right] .
$$

See Gundy and Varopoulos [10] for the rigorous statement of this equality. The interplay between the operators $\mathcal{T}_{A}^{y}$ and Riesz transforms is explained in the following theorem, consult [10] or Gundy and Silverstein [9].

Theorem 3.1 Let $A^{j}=\left[a_{\ell m}^{j}\right], j=1,2, \ldots, d$ be the $(d+1) \times(d+1)$ matrices given by

$$
a_{\ell m}^{j}= \begin{cases}1 & \text { if } \quad \ell=d+1, m=j \\ -1 & \text { if } \quad \ell=j, m=d+1 \\ 0 & \text { otherwise }\end{cases}
$$

Then $\mathcal{T}_{A^{j}}^{y} f \rightarrow R_{j} f$ almost everywhere as $y \rightarrow \infty$.

We shall require the following auxiliary fact, see Lemma 3.2 in [16].

Lemma 3.2 Let $f \in C_{0}^{\infty}\left(\mathbb{R}^{d}\right)$ and $A=A^{j}$ for some $j$. Then (3.1) holds for all $g \in L^{q}\left(\mathbb{R}^{d}\right), 1<q<\infty$. 
We are ready to establish the inequalities of Theorem 1.1 .

Proof of (1.8) and (1.9) Clearly, it suffices to establish the estimates for $R_{1}$ and $f \in C_{0}^{\infty}\left(\mathbb{R}^{d}\right)$. We will only show (1.8), the argumentation leading to the second bound is similar and left to the reader. Let $A=A^{1}$, fix $p \in[1, \infty)$ and, for a given smooth function $f: \mathbb{R}^{d} \rightarrow[0,1]$, denote $g(x)=f(x)\left|\mathcal{T}_{A}^{y} f(x)\right|^{p-2} \mathcal{T}_{A}^{y} f(x)$ (when $\mathcal{T}_{A}^{y} f(x)=0$, we set $g(x)=0$ ). It is convenient to split the reasoning into two parts.

Step 1. First we will show that $g$ belongs to $L^{r}\left(\mathbb{R}^{d}\right)$ for some $1<r<\infty$. This is clear when $p=1$, since then $|g|^{2} \leq f^{2} \leq f \in L^{1}\left(\mathbb{R}^{d}\right)$. So, suppose that $1<p<\infty$, fix $x \in \mathbb{R}$ and $y>0$. Consider the pair $\xi=\xi^{x, y}=\left(\xi_{t}\right)_{t \geq 0}, \zeta=\zeta^{x, y}=\left(\zeta_{t}\right)_{t \geq 0}$ of martingales given by

$$
\begin{aligned}
\xi_{t} & =U_{f}\left(x+X_{\tau(y) \wedge t}, y+Y_{\tau(y) \wedge t}\right) \\
& =U_{f}(x, y)+\int_{0+}^{\tau(y) \wedge t} \nabla U_{f}\left(x+X_{s}, y+Y_{s}\right) \cdot \mathrm{d}\left(X_{s}, Y_{s}\right)
\end{aligned}
$$

and

$$
\zeta_{t}=\int_{0+}^{\tau(y) \wedge t} A^{j} \nabla U_{f}\left(x+X_{s}, y+Y_{s}\right) \cdot \mathrm{d}\left(X_{s}, Y_{s}\right)
$$

for $t \geq 0$. Then the martingale $\zeta$ is orthogonal and differentially subordinate to $\xi$, since $\langle A x, x\rangle=0$ and $|A x| \leq|x|$ for all $x \in \mathbb{R}^{d}$ (see the beginning of Sect. 2). Therefore, by Theorem 2.1,

$$
\left\|\zeta_{\tau(y)}\right\|_{p}^{p}=\|\zeta\|_{p}^{p} \leq \cot ^{p}\left(\frac{\pi}{2 p^{*}}\right)\|\xi\|_{p}^{p}=\cot ^{p}\left(\frac{\pi}{2 p^{*}}\right)\left\|\xi_{\tau(y)}\right\|_{p}^{p}, \quad 1<p<\infty
$$

Integrating both sides with respect to $x \in \mathbb{R}^{d}$ gives

$$
\begin{aligned}
\int_{\mathbb{R}^{d}} \mathbb{E}|A * f(x, y)|^{p} \mathrm{~d} x & \leq \cot ^{p}\left(\frac{\pi}{2 p^{*}}\right) \int_{\mathbb{R}^{d}} \mathbb{E}\left|f\left(x+X_{\tau(y)}\right)\right|^{p} \mathrm{~d} x \\
& =\cot ^{p}\left(\frac{\pi}{2 p^{*}}\right)\|f\|_{L^{p}\left(\mathbb{R}^{d}\right)}^{p}
\end{aligned}
$$

by virtue of Fubini's theorem. In addition, for any $h \in L^{q}\left(\mathbb{R}^{d}\right)(q=p /(p-1)$ is the harmonic conjugate to $p$ ),

$$
\int_{\mathbb{R}^{d}} \mathbb{E}\left|h\left(x+X_{\tau(y)}\right)\right|^{q} \mathrm{~d} x=\|h\|_{L^{q}\left(\mathbb{R}^{d}\right)}^{q} .
$$


Combining these estimates with (3.1) and Hölder's inequality yields

$$
\begin{aligned}
\left|\int_{\mathbb{R}^{d}} \mathcal{T}_{A}^{y} f(x) h(x) \mathrm{d} x\right| & =\left|\int_{\mathbb{R}^{d}} \mathbb{E}\left[A * f(x, y) h\left(x+X_{\tau(y)}\right)\right] \mathrm{d} x\right| \\
& \leq \cot ^{p}\left(\frac{\pi}{2 p^{*}}\right)\|f\|_{L^{p}\left(\mathbb{R}^{d}\right)}\|h\|_{L^{q}\left(\mathbb{R}^{d}\right)},
\end{aligned}
$$

which implies that $\mathcal{T}_{A}^{y} f \in L^{p}\left(\mathbb{R}^{d}\right)$. This, in turn, gives $g \in L^{p /(p-1)}\left(\mathbb{R}^{d}\right)$, since $f \in[0,1]$.

Step 2. By the previous step and Lemma 3.2, we have

$$
\begin{aligned}
\int_{\mathbb{R}^{d}} f(x)\left|\mathcal{T}_{A}^{y} f(x)\right|^{p} \mathrm{~d} x & =\int_{\mathbb{R}^{d}} \mathcal{T}_{A}^{y} f(x) g(x) \mathrm{d} x \\
& =\int_{\mathbb{R}^{d}} \mathbb{E}\left[A * f(x, y) g\left(x+X_{\tau(y)}\right)\right] \mathrm{d} x \\
& \leq \int_{\mathbb{R}^{d}} \mathbb{E}|A * f(x, y)|^{p} f\left(x+X_{\tau(y)}\right) \mathrm{d} x .
\end{aligned}
$$

The latter passage is obvious when $p=1$, while for remaining values of $p$ follows quickly from Hölder's inequality. Introduce the martingales $\xi^{x, y}$ and $\zeta^{x, y}$ as in Step 1. By (2.1), we get that

$$
\begin{aligned}
\int_{\mathbb{R}^{d}} \mathbb{E}|A * f(x, y)|^{p} f\left(x+X_{\tau(y)}\right) \mathrm{d} x & =\int_{\mathbb{R}^{d}} \mathbb{E} \xi_{\infty}^{x, y}\left|\zeta_{\infty}^{x, y}\right|^{p} \mathrm{~d} x \\
& \leq \int_{\mathbb{R}^{d}} U_{1}\left(\left\|\xi^{x, y}\right\|_{1}, 0\right) \mathrm{d} x
\end{aligned}
$$

The function $U_{1}$ is nonnegative, so by Fubini's theorem, we may write

$$
\begin{aligned}
& \int_{\mathbb{R}^{d}} U_{1}\left(\left\|\xi^{x, y}\right\|_{1}, 0\right) \mathrm{d} x \\
& \quad=\int_{0}^{\infty} \frac{\left|\frac{1}{\pi} \log \right| s||^{p}}{(s+1)^{2}} \int_{\mathbb{R}^{d}} \frac{\sin \left(\pi\left\|\xi^{x, y}\right\|_{1}\right)}{\pi\left\|\xi^{x, y}\right\|_{1}} \frac{(s+1)^{2}}{s^{2}+2 s \cos \left(\pi\left\|\xi^{x, y}\right\|_{1}\right)+1}\left\|\xi^{x, y}\right\|_{1} \mathrm{~d} x \mathrm{~d} s .
\end{aligned}
$$

Since $f$ is nonnegative and integrable, we have

$$
\lim _{y \rightarrow \infty} \sup _{x \in \mathbb{R}}\left\|\xi^{x, y}\right\|_{1}=\lim _{y \rightarrow \infty} \sup _{x \in \mathbb{R}} U_{f}(x, y)=0 .
$$

Furthermore, an application of Fubini's theorem gives 


$$
\int_{\mathbb{R}^{d}}\left\|\xi^{x, y}\right\|_{1} \mathrm{~d} x=\|f\|_{L^{1}\left(\mathbb{R}^{d}\right)}
$$

Thus, by Lebesgue's dominated convergence theorem, Fatou's lemma and Theorem 3.1 , we get the bound

$$
\int_{\mathbb{R}^{d}} f(x)\left|R_{1} f(x)\right|^{p} \mathrm{~d} x \leq \frac{1}{\pi^{p}} \int_{0}^{\infty} \frac{(\log s)^{p}}{(s+1)^{2}} \mathrm{~d} s \cdot\|f\|_{L^{1}\left(\mathbb{R}^{d}\right)} .
$$

It remains to note that

$$
\begin{aligned}
\frac{1}{\pi^{p}} \int_{0}^{\infty} \frac{|\log s|^{p}}{(s+1)^{2}} \mathrm{~d} s & =\frac{1}{\pi^{p}} \int_{\mathbb{R}} \frac{|t|^{p}}{\left(e^{t}+1\right)^{2}} e^{t} \mathrm{~d} t \\
& =\frac{1}{\pi^{p}} \int_{0}^{\infty} \frac{t^{p} e^{t}}{\left(e^{t}+1\right)^{2}} \mathrm{~d} t+\frac{1}{\pi^{p}} \int_{0}^{\infty} \frac{t^{p} e^{-t}}{\left(e^{-t}+1\right)^{2}} \mathrm{~d} t \\
& =\frac{2}{\pi^{p}} \int_{0}^{\infty} \frac{t^{p} e^{t}}{\left(e^{t}+1\right)^{2}} \mathrm{~d} t=\frac{2}{\pi^{p}} \int_{0}^{\infty} \frac{p t^{p-1}}{e^{t}+1} \mathrm{~d} t \\
& =2 \int_{0}^{\infty} \frac{p t^{p-1}}{e^{\pi t}+1} \mathrm{~d} t,
\end{aligned}
$$

so (1.8) follows.

\section{Sharpness}

If $d=1$ and $f=\chi_{E}$ for some $E$ of finite and nonzero Lebesgue measure, then equality holds in (1.10). This follows immediately from the identity (1.2). In the case $d>1$, we use a well-known transference-type argument, see e.g. [16] for a similar reasoning. As previously, we focus on the sharpness of (1.10), which will in turn imply the optimality of the constants appearing in (1.8) and (1.9). Clearly, it is enough to deal with the Riesz transform $R_{1}$ only. Suppose that for a fixed $p \in[1, \infty)$ we have

$$
\int_{\mathbb{R}}\left|R_{1} \chi_{E}(x)\right|^{p} \mathrm{~d} x \leq C|E|
$$

for all Borel subsets $E$ of $\mathbb{R}^{d}$. For $t>0$, define the dilation operator $\delta_{t}$ as follows: for any function $g: \mathbb{R} \times \mathbb{R}^{d-1} \rightarrow \mathbb{R}$, we let $\delta_{t} g(\xi, \zeta)=g(\xi, t \zeta)$; for any $A \subset \mathbb{R} \times \mathbb{R}^{d-1}$, let $\delta_{t} A=\{(\xi, t \zeta):(\xi, \zeta) \in A\}$. By (4.1), the operator $T_{t}:=\delta_{t}^{-1} \circ R_{1} \circ \delta_{t}$ satisfies 


$$
\begin{aligned}
\int_{\mathbb{R}^{d}}\left|T_{t} \chi_{E}(x)\right|^{p} \mathrm{~d} x & =t^{d-1} \int_{\mathbb{R}^{d}}\left|R_{1} \chi_{\delta_{t}^{-1} E}\right|^{p} \mathrm{~d} x \\
& \leq C t^{d-1}\left|\delta_{t}^{-1} E\right|=C|E| .
\end{aligned}
$$

It is straightforward to check that the Fourier transform $\mathcal{F}$ satisfies the identity $\mathcal{F}=$ $t^{d-1} \delta_{t} \circ \mathcal{F} \circ \delta_{t}$, so the operator $T_{t}$ is a Fourier multiplier such that

$$
\widehat{T_{t} f}(\xi, \zeta)=-i \frac{\xi}{\left(\xi^{2}+t^{2}|\zeta|^{2}\right)^{1 / 2}} \widehat{f}(\xi, \zeta), \quad(\xi, \zeta) \in \mathbb{R} \times \mathbb{R}^{d-1}
$$

for $f \in L^{2}\left(\mathbb{R}^{d}\right)$. By Lebesgue's dominated convergence theorem, we have

$$
\lim _{t \rightarrow 0} \widehat{T_{t} f}(\xi, \zeta)=\widehat{T_{0} f}(\xi, \zeta)
$$

in $L^{2}\left(\mathbb{R}^{d}\right)$, where

$$
\widehat{T_{0} f}(\xi, \zeta)=-i \operatorname{sgn}(\xi) \widehat{f}(\xi, \zeta)
$$

Combining this with Plancherel's theorem, we conclude that there is a sequence $\left(t_{n}\right)_{n \geq 1}$ decreasing to 0 such that $T_{t_{n}} f$ converges to $T_{0} f$ almost everywhere. Thus, taking $f=\chi_{E}$ and applying Fatou's lemma together with (4.2), we obtain

$$
\int_{\mathbb{R}^{d}}\left|T_{0} \chi_{E}(x)\right| \mathrm{d} x \leq C|E| .
$$

Now pick $E=B \times[0,1]^{d-1}$, where $B$ is a Borel subset of $\mathbb{R}$ with $0<|B|<\infty$. Then $T_{0} \chi_{E}(\xi, \zeta)=\mathcal{H} \chi_{B}(\xi) \chi_{[0,1]^{d-1}}(\zeta)$, because of the identity (4.3),

$$
\widehat{T_{0} \chi_{E}}(\xi, \zeta)=-i \operatorname{sgn}(\xi) \widehat{\chi_{B}}(\xi) \widehat{\chi_{[0,1]^{d}-1}}(\zeta)
$$

Plugging this into (4.4) and integrating out the variable $\zeta$, we obtain

$$
\int_{\mathbb{R}}\left|\mathcal{H} \chi_{B}(\xi)\right|^{p} \mathrm{~d} \xi \leq C|B|,
$$

which implies $C \geq 2 \int_{0}^{\infty} \frac{p t^{p-1}}{\sinh (\pi t)} \mathrm{d} t$ by the case $d=1$. The proof is complete.

Acknowledgments The author would like to thank an anonymous referee for the careful reading of the paper and many helpful remarks. The research was partially supported by Polish Ministry of Science and Higher Education (MNiSW) grant IP2011 039571 'Iuventus Plus'.

Open Access This article is distributed under the terms of the Creative Commons Attribution License which permits any use, distribution, and reproduction in any medium, provided the original author(s) and the source are credited. 


\section{References}

1. Bañuelos, R., Wang, G.: Sharp inequalities for martingales with applications to the Beurling-Ahlfors and Riesz transformations. Duke Math. J. 80, 575-600 (1995)

2. Bañuelos, R., Wang, G.: Sharp inequalities for martingales under orthogonality and differential subordination. Ill. J. Math. 40, 678-691 (1996)

3. Birman, M.S.: Re-expansion operators as objects of spectral analysis. In: Linear and Complex Analysis Problem Book, Lecture Notes in Mathematics, vol. 1043, pp. 130-134. Springer, Berlin (1984)

4. Burkholder, D.L.: Explorations in Martingale Theory and Its Applications. École d'Ete de Probabilités de Saint-Flour XIX-1989, Lecture Notes in Mathematics. Springer, Berlin (1991)

5. Calderón, A.P.: Singular integrals. Bull Am. Math. Soc. 72, 427-465 (1966)

6. Colzani, L., Laeng, E., Monzón, L.: Variations on a theme of Boole and Stein-Weiss. J. Math. Anal. Appl. 363, 225-229 (2010)

7. Dellacherie, C., Meyer, P.-A.: Probabilities and Potential B: Theory of Martingales. North Holland, Amsterdam (1982)

8. Gokhberg, I., Krupnik, N.: Introduction to the Theory of One-Dimensional Singular Integral Operators (in Russian) Izdat. "Štiinca", Kishinev (1973)

9. Gundy, R.F., Silverstein, M.: On a Probabilistic Interpretation for Riesz Transforms, Lecture Notes in Mathematics, vol. 923. Springer, Berlin (1982)

10. Gundy, R.F., Varopoulos, N.Th: Les transformations de Riesz et les integrales stochastiques. C. R. Acad. Sci. Paris Sér. A-B 289, A13-A16 (1979)

11. Hollenbeck, B., Kalton, N.J., Verbitsky, I.E.: Best constants for some operators associated with the Fourier and Hilbert transforms. Studia Math. 157, 237-278 (2003)

12. Il'in, E.M.: Scattering characteristics of a problem of diffraction by a wedge and by a screen (in Russian). Investigations on linear operators and the theory of functions, X. Zap. Nauchn. Sem. Leningrad. Otdel. Mat. Inst. Steklov. (LOMI) 107, 193-197 (1982)

13. Il'in, E.M.: The scattering matrix for a problem of diffraction by a wedge (in Russian). In: Operator Theory and Function Theory, No. 1, Leningrad University, pp. 87-100 (1983)

14. Laeng, E.: On the $L^{p}$ norms of the Hilbert transform of a characteristic function. J. Funct. Anal. 262, 4534-4539 (2012)

15. Osękowski, A.: On the best constants in the weak type inequalities for re-expansion operator and Hilbert transform. Trans. Am. Math. Soc. 364, 4303-4322 (2012)

16. Osȩkowski, A.: Sharp logarithmic inequalities for Riesz transforms. J. Funct. Anal. 263, 89-108 (2012)

17. Osȩkowski, A.: Sharp Martingale and Semimartingale Inequalities. Birkhäuser, Heidelberg (2012)

18. Stein, E.M.: Singular Integrals and Differentiability Properties of Functions. Princeton University Press, Princeton (1970)

19. Stein, E.M., Wiesz, G.: An extension of a theorem of Marcinkiewicz and some of its applications. J. Math. Mech. 8, 263-284 (1959)

20. Stein, E.M., Wiesz, G.: Introduction to Fourier Analysis on Euclidean Spaces. Princeton University Press, Princeton (1971)

21. Wang, G.: Differential subordination and strong differential subordination for continuous time martingales and related sharp inequalities. Ann. Probab. 23, 522-551 (1995) 